\title{
Particle ratios from AGS to RHIC in an interacting hadronic model
}

\author{
D Zschiesche ${ }^{1}$, G Zeeb ${ }^{1}$, K Paech ${ }^{1}$, S Schramm ${ }^{2}$ and H Stöcker ${ }^{3,4}$ \\ ${ }^{1}$ Institut für Theoretische Physik, Robert Mayer Str. 8-10, D-60054 Frankfurt am Main, Germany \\ 2 Argonne National Laboratory, 9700 S. Cass Avenue, Argonne, IL 60439, USA \\ ${ }^{3}$ Institut für Theoretische Physik, Robert Mayer Str. 8-10, D-60054 Frankfurt am Main, Germany \\ ${ }^{4}$ Laboratoire de Physique Subatomique et des Technologies Associées, University of Nantes - \\ IN2P3/CNRS - Ecole des Mines de Nantes 4 rue Alfred Kastler, F-44072 Nantes Cedex 03, \\ France
}

Received 2 June 2003

Published 11 December 2003

Online at stacks.iop.org/JPhysG/30/S381 (DOI: 10.1088/0954-3899/30/1/046)

\begin{abstract}
The measured particle ratios in central heavy-ion collisions at RHIC-BNL are investigated within a chemical and thermal equilibrium chiral $S U$ (3) $\sigma-\omega$ approach. The commonly adopted non-interacting gas calculations yield temperatures close to or above the critical temperature for the chiral phase transition, but without taking into account any interactions. In contrast, the chiral $S U$ (3) model predicts temperature and density dependent effective hadron masses and effective chemical potentials in the medium and a transition to a chirally restored phase at high temperatures or chemical potentials. Three different parametrizations of the model, which show different types of phase transition behaviour, are investigated. We show that if a chiral phase transition occured in those collisions, 'freezing' of the relative hadron abundances in the symmetric phase is excluded by the data. Therefore, either very rapid chemical equilibration must occur in the broken phase, or the measured hadron ratios are the outcome of the dynamical symmetry breaking. Furthermore, the extracted chemical freeze-out parameters differ considerably from those obtained in simple non-interacting gas calculations. In particular, the three models yield up to $35 \mathrm{MeV}$ lower temperatures than the free gas approximation. The inmedium masses turn out to differ up to $150 \mathrm{MeV}$ from their vacuum values.
\end{abstract}

\section{Introduction}

Thermodynamical equilibrium calculations of particle production in high energy particle- and nuclear collisions have been carried out for a long time [1-11]. Recently hadron abundances and particle ratios have been measured in heavy-ion collisions from SIS, AGS, SPS to RHIC energies. These data have revived the interest in the extraction of temperatures and chemical potentials from thermal equilibrium 'chemical' model analyses. The experimentally 
determined hadron ratios can be fitted well with straightforward non-interacting gas model calculations [7-13], if a sudden breakup of a thermalized source is assumed and once the subsequent feeding of the various channels by the strongly decaying resonances is taken into account. From the $\chi^{2}$ freeze-out fits one has constructed a quite narrow band of freeze-out values in the $T-\mu_{B}$ plane (see, e.g. $[12,13]$ ). The extracted freeze-out parameters are fairly close to the phase transition curve for SPS and RHIC energies. However, when we are indeed so close to the phase transition or to a crossover as suggested by the data for $T$ and $\mu_{B}$, we cannot afford to neglect the very in-medium effects we are after, and which, after all, do produce the phase transition. Thus, since non-interacting gas models neglect any kind of possible in-medium modifications they cannot yield information about the phase transition.

Therefore, we will employ below a relativistic selfconsistent chiral model of hadrons and hadron matter developed in [14-16]. This model can be used as a thermodynamically consistent effective theory or as a toy model, which embodies the restoration of chiral symmetry at high temperatures or densities. Therefore, the model predicts temperature and density dependent hadronic masses and effective chemical potentials, which have already been proposed and considered in [4, 16-20]. Thus, using the chiral $S U(3)$ model we can investigate, whether the freeze-out in fact takes place close to the phase transition boundary (if it exists) and if the extracted $T, \mu_{B}$ parameters are strongly model dependent. Depending on the chosen parameters and degrees of freedom different scenarios for the chiral phase change are predicted by the model: Strong or weak first-order phase transition or a crossover. The transitions take place around $T_{c}=155 \mathrm{MeV}[16,21]$, which is in qualitative agreement with lattice predictions [22] for the critical temperature for the onset of a deconfined phase which coincides with that of a chirally restored phase [23].

\section{Model description}

The chiral $S U(3)$ model is presented in detail in $[15,16]$. We will briefly introduce the model here: we consider a relativistic field theoretical model of baryons and mesons built on chiral symmetry and broken scale invariance. The general form of the Lagrangean looks as follows:

$$
\mathcal{L}=\mathcal{L}_{\text {kin }}+\sum_{W=X, Y, V, \mathcal{A}, u} \mathcal{L}_{\mathrm{BW}}+\mathcal{L}_{\mathrm{VP}}+\mathcal{L}_{\mathrm{vec}}+\mathcal{L}_{0}+\mathcal{L}_{\mathrm{SB}}
$$

$\mathcal{L}_{\text {kin }}$ is the kinetic energy term, $\mathcal{L}_{\mathrm{BW}}$ includes the interaction terms of the different baryons with the various spin-0 and spin-1 mesons (see [15] for details). The baryon masses are generated by both, the non-strange $\sigma(\langle\mathrm{q} \overline{\mathrm{q}}\rangle)$ and the strange $\zeta(\langle\mathrm{s} \overline{\mathrm{s}}\rangle)$ scalar condensate. $\mathcal{L}_{\mathrm{VP}}$ contains the interaction terms of vector mesons with pseudoscalar mesons. $\mathcal{L}_{\text {vec }}$ generates the masses of the spin- 1 mesons through interactions with spin- 0 fields, and $\mathcal{L}_{0}$ gives the meson-meson interaction terms which induce the spontaneous breaking of chiral symmetry. It also includes a scale-invariance breaking logarithmic potential. Finally, $\mathcal{L}_{\mathrm{SB}}$ introduces an explicit symmetry breaking of the $U(1)_{A}$, the $S U(3)_{V}$, and the chiral symmetry. All these terms have been discussed in detail in $[15,16]$.

The hadronic matter properties at finite density and temperature are studied in the meanfield approximation [24]. Then the Lagrangean (1) becomes

$$
\begin{aligned}
& \mathcal{L}_{B X}+\mathcal{L}_{B V}=-\sum_{i} \bar{\psi}_{i}\left[g_{i \omega} \gamma_{0} \omega^{0}+g_{i \phi} \gamma_{0} \phi^{0}+m_{i}^{*}\right] \psi_{i} \\
& \mathcal{L}_{\mathrm{vec}}=\frac{1}{2} m_{\omega}^{2} \frac{\chi^{2}}{\chi_{0}^{2}} \omega^{2}+\frac{1}{2} m_{\phi}^{2} \frac{\chi^{2}}{\chi_{0}^{2}} \phi^{2}+g_{4}^{4}\left(\omega^{4}+2 \phi^{4}\right)
\end{aligned}
$$




$$
\begin{gathered}
\mathcal{V}_{0}=\frac{1}{2} k_{0} \chi^{2}\left(\sigma^{2}+\zeta^{2}\right)-k_{1}\left(\sigma^{2}+\zeta^{2}\right)^{2}-k_{2}\left(\frac{\sigma^{4}}{2}+\zeta^{4}\right)-k_{3} \chi \sigma^{2} \zeta \\
+k_{4} \chi^{4}+\frac{1}{4} \chi^{4} \ln \frac{\chi^{4}}{\chi_{0}^{4}}-\frac{\delta}{3} \chi^{4} \ln \frac{\sigma^{2} \zeta}{\sigma_{0}^{2} \zeta_{0}} \\
\mathcal{V}_{S B}=\left(\frac{\chi}{\chi_{0}}\right)^{2}\left[m_{\pi}^{2} f_{\pi} \sigma+\left(\sqrt{2} m_{K}^{2} f_{K}-\frac{1}{\sqrt{2}} m_{\pi}^{2} f_{\pi}\right) \zeta\right],
\end{gathered}
$$

where $m_{i}$ is the effective mass of the hadron species $i$. $\sigma$ and $\zeta$ correspond to the scalar condensates, $\omega$ and $\phi$ represent the non-strange and the strange vector field respectively, and $\chi$ is the scalar-isoscalar dilaton field, which mimics the effects of the gluon condensate [25]. Only the scalar $\left(\mathcal{L}_{B X}\right)$ and the vector meson terms $\left(\mathcal{L}_{B V}\right)$ contribute to the baryonmeson interaction, since for all other mesons the expectation value vanishes in the mean-field approximation. The grand canonical potential $\Omega$ per volume $V$ as a function of chemical potential $\mu$ and temperature $T$ can be written as:

$\frac{\Omega}{V}=-\mathcal{L}_{\text {vec }}-\mathcal{L}_{0}-\mathcal{L}_{S B}-\mathcal{V}_{\text {vac }} \mp T \sum_{i} \frac{\gamma_{i}}{(2 \pi)^{3}} \int \mathrm{d}^{3} k\left[\ln \left(1 \pm \mathrm{e}^{-\frac{1}{T}\left[E_{i}^{*}(k)-\mu_{i}^{*}\right]}\right)\right]$

with the baryons (top sign) and mesons (bottom sign). The vacuum energy $\mathcal{V}_{\text {vac }}$ (the potential at $\rho_{B}=0, T=0$ ) has been subtracted in order to get a vanishing vacuum energy. $\gamma_{i}$ denote the hadronic spin-isospin degeneracy factors. The single particle energies are $E_{i}^{*}(k)=\sqrt{k_{i}^{2}+m_{i}^{* 2}}$ and the effective chemical potentials read $\mu_{i}^{*}=\mu_{i}-g_{i \omega} \omega-g_{\phi i} \phi$.

The mesonic fields are determined by extremizing $\frac{\Omega}{V}(\mu, T=0)$. The density of particle $i$ can be calculated by differentiating $\Omega$ with respect to the corresponding chemical potential $\mu_{i}$. This yields

$$
\rho_{i}=\gamma_{i} \int \frac{\mathrm{d}^{3} k}{(2 \pi)^{3}}\left[\frac{1}{\exp \left[\left(E_{i}^{*}-\mu_{i}^{*}\right) / T\right] \pm 1}\right]
$$

All other thermodynamic quantities can also be obtained from the grand canonical potential. In the present calculation the lowest lying baryonic octet and decuplet and the lowest lying mesonic nonets are coupled to the relativistic mean fields. Depending on the coupling of the baryon resonances (the decuplet) to the field equations, the model shows a first-order phase transition or a crossover (for details see [21]). We will use three different parameter sets: parameter set CI treats the members of the baryon decuplet as free particles, which yields a crossover behaviour. Parameter sets CII and CIII include also the (anti)-baryon decuplet as sources for the meson field equations. They differ by an additional explicit symmetry breaking for the baryon resonances along the hypercharge direction, as described in [15] for the baryon octet. This is included in CII and not used in CIII. This leads to a weak first-order phase transition at $\mu=0$ for CII and two first-order phase transitions for CIII, which can be viewed as one strong first-order phase transition. Heavier resonances up to $m=2 \mathrm{GeV}$ are always included as free particles (for details see [21]). The critical energy densities, the entropy densities and the transition temperatures for $\mu_{q}=\mu_{s}=0\left(\mu_{q}=\mu_{B} / 3, \mu_{s}=\mu_{B} / 3-\mu_{S}\right)$ are specified in table 1.

\section{Particle ratios in the chiral $S U(3) \times S U(3)$ model}

Since the chiral $S U(3)$ model predicts density and temperature-dependent hadronic masses and effective potentials, in contrast to non-interacting models, the resulting particle ratios and therefore the deduced freeze-out temperatures and baryon chemical potentials are expected to change [26]. Hence in the following, we identify combinations of temperatures and chemical 
Table 1. Energy density, entropy density and phase transition temperatures for CII, CIII, $\mu_{q}=\mu_{s}=0$. The $(-),(+)$ signs refer to an approach to the phase transition from below and above, respectively. $T_{c}$ denotes the phase transition temperature. $\epsilon_{0}=138.45 \mathrm{MeV} \mathrm{fm}^{-3}$ denotes the energy density of nuclear matter in the ground state.

\begin{tabular}{lcclcl}
\hline & $\epsilon^{-} / \epsilon_{0}$ & $\epsilon^{+} / \epsilon_{0}$ & $s^{-}\left(\mathrm{fm}^{-3}\right)$ & $s^{+}\left(\mathrm{fm}^{-3}\right)$ & $T_{c}(\mathrm{MeV})$ \\
\hline CII & 2.8 & 7.2 & 2.8 & 6.7 & 156.3 \\
CIII (1st PT) & 2.3 & 8.3 & 2.4 & 7.9 & 153.4 \\
CIII (2nd PT) & 10.5 & 17.1 & 9.8 & 15.7 & 155.5 \\
\hline
\end{tabular}

potentials that fit the observed particle ratios in the chiral model. In all calculations the value of the strange chemical potential $\mu_{S}$ is chosen such that the net strangeness $f_{s}=0$. We are looking for minima of $\chi^{2}$ with

$$
\chi^{2}=\sum_{i} \frac{\left(r_{i}^{\exp }-r_{i}^{\text {model }}\right)^{2}}{\sigma_{i}^{2}} .
$$

Here $r_{i}^{\exp }$ is the experimental ratio, $r_{i}^{\text {model }}$ is the ratio calculated in the model and $\sigma_{i}$ represents the error in the experimental data points. At RHIC, we use the same ratios as in [11]: $\overline{\mathrm{p}} / \mathrm{p}, \bar{\Lambda} / \Lambda, \bar{\Xi} / \Xi, \pi^{-} / \pi^{+}, \mathrm{K}^{-} / \mathrm{K}^{+}, \mathrm{K}^{-} / \pi^{-}, \mathrm{K}_{0}^{*} / \mathrm{h}^{-}, \overline{\mathrm{K}}_{0}^{*} / \mathrm{h}^{-}$. For SPS $158 \mathrm{~A} \mathrm{GeV}$ the following ratios were used: $\mathrm{K}^{+} / \mathrm{K}^{-}, \mathrm{K}^{+} /\left(\pi^{+}+\pi^{-}\right), \bar{\Lambda} / \Lambda, \Xi / \bar{\Xi}, \bar{\Omega} / \Omega, \bar{\Xi} / \Lambda, \Omega / \bar{\Xi}^{-}$, $\Lambda /\left(\pi^{+}+\pi^{-}\right)$. For SPS $40 \mathrm{~A} \mathrm{GeV:} \mathrm{K}^{+} / \mathrm{K}^{-}, \mathrm{K}^{+} /\left(\pi^{+}+\pi^{-}\right), \bar{\Lambda} / \Lambda, \Lambda /\left(\pi^{+}+\pi^{-}\right)$. And for AGS 11.6 $A \mathrm{GeV}$ the considered ratios are: $\mathrm{K}^{+} / \pi^{+}, \mathrm{K}^{+} / \mathrm{K}^{-}, \Lambda / \pi^{+}, \mathrm{p} / \pi^{+}, \overline{\mathrm{p}} / \mathrm{p}$ taken from [10].

Even though the only parameters in a thermal and chemical equilibrium approach on first sight are the temperature and the baryon chemical potential, there exist further unknowns: On the one hand, some decays of high mass resonances are not well known and on the other hand, the effect of weak decays in the experiments strongly depends on the detector geometry and on the reconstruction efficiency of the experiments. The feeding correction from the strong and electromagnetic decays of the hadronic resonances used here employs the procedure used in the UrQMD model $[27,28]$. Weak decays are not considered here. We rather focus on the principal question whether an interacting chiral $S U(3)$ approach with $m^{*} \neq m_{\text {vac }}$ can at all describe the particle yields at RHIC. Fine tuning of the $\chi^{2}$ by adjustment of the weak decay scheme is not our intention. Even though it has been shown [29] that $\chi^{2}$ values may be improved by including weak decays.

To compare the quality of the fits obtained in the chiral model with those obtained from the non-interacting gas approach, we set all masses and chemical potentials contained in the chiral model to their vacuum values and again use the same UrQMD feeding procedure as for the interacting model. This yields the ideal gas denoted $i g_{F F M}$. We find that the resulting ideal gas ratios are not identical but comparable to those obtained in the literature [11, 26, 29, 30]. The differences should only result from a different treatment of weak interactions and from the uncertainty in the decay scheme of high mass resonances.

\section{Results for Au+Au collisions at RHIC}

First, we find that a reasonable fit of the measured particle ratios at RHIC is possible in all three phase transition scenarios of the chiral model and the ideal gas case with comparable quality.

Second, the resulting freeze-out values depend on the model employed, i.e. crossover, weak first-order, strong first-order or free hadron gas. 

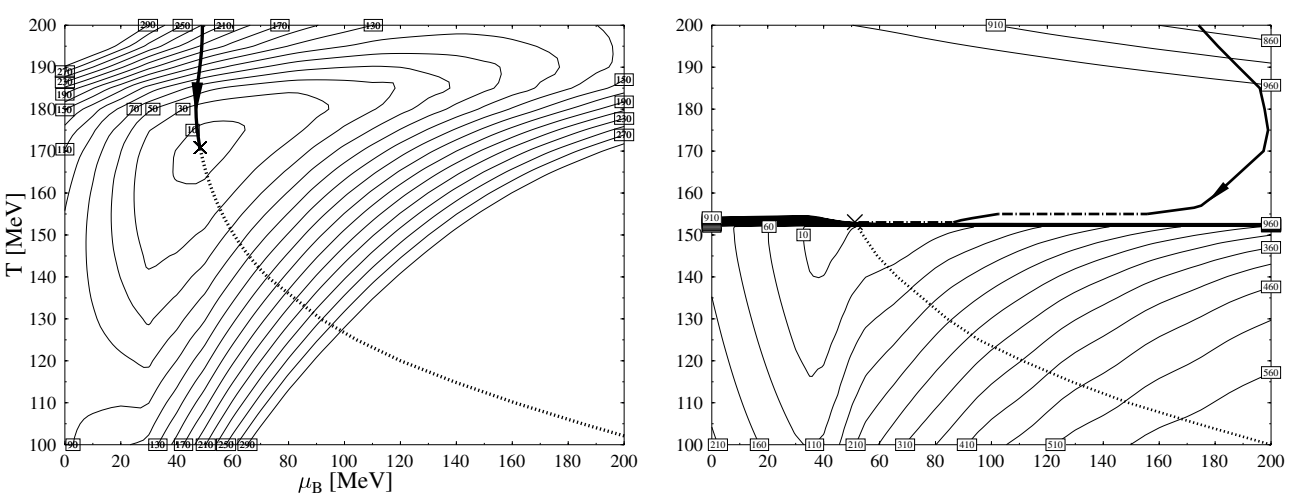

Figure 1. $\chi^{2}$ contours in the $T-\mu_{B}$ plane for CI (left) and CIII (right). Data are taken from [11]. On the left, the adiabatic path (constant entropy per net baryon $S / A$ ), corresponding to expanisve cooling of an ideal fluid, is also shown. $\mu_{S}$ is chosen such that $f_{s}=0$.
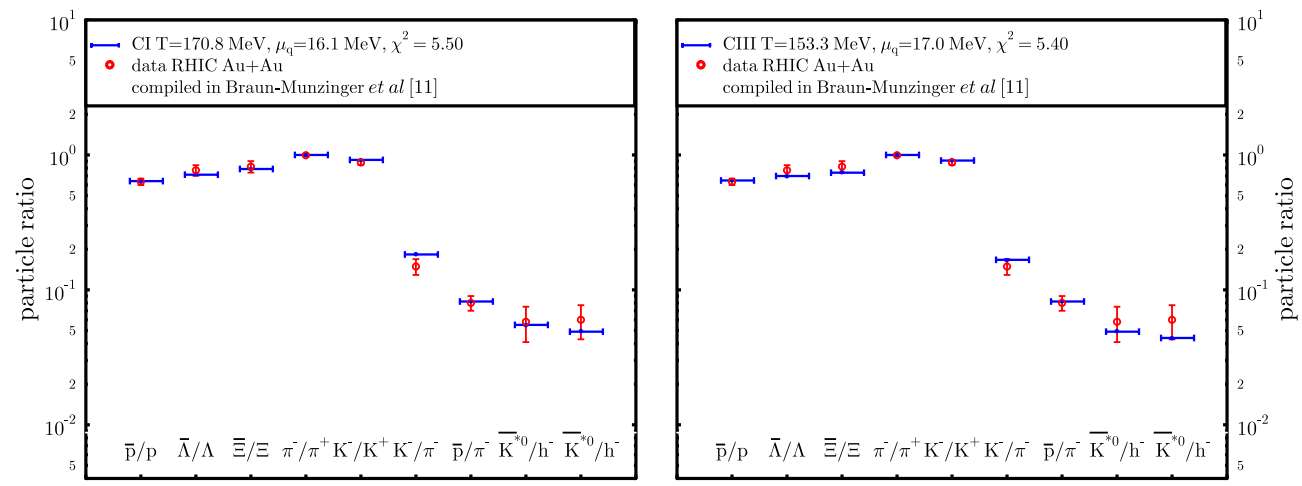

Figure 2. Particle ratios calculated with $\mathrm{CI}$ (left) and CIII (right) compared to RHIC data as compiled in [11].

Third, a reasonable description of the data is impossible above $T_{c}$ in the models showing a first-order phase transition. This shows that no direct freeze-out from the restored phase is observed.

Figure 1 shows the value of $\chi^{2}$ in the $T-\mu_{B}$ plane for the crossover case and for the strong first-order phase transition. We see that the best fit $T-\mu_{B}$ values differ in both models. Furthermore, in the crossover case $\chi^{2}$ is well behaved as a function of $T$ and $\mu_{B}$. In contrast, the model with a strong first-order phase transition shows a very steep increase of $\chi^{2}$ at the phase transition boundary: the quality of the fit decreases drastically due to the jump of the effective masses at the phase transition boundary. Above $T_{c}$ the $\chi^{2}$ values are inacceptable, $\chi^{2}>500$.

The resulting best-fit particle ratios, $\chi^{2}$-values and thermodynamic quantities are shown in table 2 and figure 2.

The $\chi^{2}$ values for the chiral model are: $\chi_{\mathrm{CI}}^{2}=5.50, \chi_{\mathrm{CII}}^{2}=5.73$ and $\chi_{\mathrm{CIII}}^{2}=5.40$. Thus, all three parameter sets describe the data equally well. Furthermore, the agreement is as good as in the non-interacting gas calculation $\left(\chi_{i g}^{2}=5.72[11], \chi_{F F M}^{2}=5.66\right)$. The best fit $T-\mu_{B}$ parameters vary quite considerably between the different models. The non-interacting gas calculation yields $T=187.6 \mathrm{MeV}$ and $\mu_{B}=44.1 \mathrm{MeV}$. These freeze-out values can be 
Table 2. Chiral fit of the particle ratios measured at RHIC at $\sqrt{s}=130 \mathrm{GeV}(\mathrm{S}=\mathrm{STAR}, \mathrm{PHE}=$ PHENIX, PHO = PHOBOS, B = BRAHMS).

\begin{tabular}{|c|c|c|c|c|c|c|}
\hline $\mathrm{Au}+\mathrm{Au}$ & Experiment & CI & CII & CIII & $i g_{F F M}$ & BMRS \\
\hline$T_{\text {chem }}(\mathrm{MeV})$ & & 170.8 & 155.0 & 153.3 & 187.6 & 174.0 \\
\hline$\mu_{\mathrm{chem}}^{B}(\mathrm{MeV})$ & & 48.3 & 54.6 & 51.0 & 44.1 & 46.0 \\
\hline$\mu_{\text {chem }}^{s}(\mathrm{MeV})$ & & 11.1 & 9.8 & 9.4 & 13.5 & 13.6 \\
\hline$\chi^{2}$ & & 5.5 & 5.7 & 5.4 & 5.7 & 5.7 \\
\hline$\rho_{\text {had }}\left(\mathrm{fm}^{-3}\right)$ & & 0.66 & 0.38 & 0.35 & 1.12 & \\
\hline$\rho_{B}+\rho_{\bar{B}}\left(\mathrm{fm}^{-3}\right)$ & & 0.15 & 0.08 & 0.07 & 0.28 & \\
\hline$p\left(\mathrm{MeV} \mathrm{fm}^{-3}\right)$ & & 108 & 55 & 51 & 207 & \\
\hline$\epsilon\left(\mathrm{MeV} \mathrm{fm}^{-3}\right)$ & & 695 & 356 & 326 & 1324 & \\
\hline$E / A(\mathrm{MeV})$ & & 1053 & 937 & 931 & 1182 & $\approx 1100$ \\
\hline$S / A$ & & 157 & 164 & 177 & 142 & \\
\hline$\overline{\mathrm{p}} / \mathrm{p}$ & $\begin{array}{l}0.65(7)[\mathrm{S}], 0.64(8)[\mathrm{PHE}] \\
0.60(7)[\mathrm{PHO}], 0.61(6)[\mathrm{B}]\end{array}$ & 0.640 & 0.648 & 0.652 & 0.629 & 0.629 \\
\hline $\bar{\Lambda} / \Lambda$ & $0.77(7)[S]$ & 0.714 & 0.695 & 0.702 & 0.721 & 0.753 \\
\hline $\bar{\Xi} / \Xi$ & $0.82(8)[\mathrm{S}]$ & 0.787 & 0.731 & 0.743 & 0.834 & 0.894 \\
\hline$\pi^{-} / \pi^{+}$ & $1.00(2)[\mathrm{PHO}], 0.95(6)[\mathrm{B}]$ & 1.000 & 1.000 & 1.000 & 1.000 & 1.007 \\
\hline $\mathrm{K}^{-} / \mathrm{K}^{+}$ & $\begin{array}{l}0.88(5)[\mathrm{S}], 0.78(13)[\mathrm{PHE}] \\
0.91(9)[\mathrm{PHO}], 0.89(7)[\mathrm{B}]\end{array}$ & 0.919 & 0.914 & 0.915 & 0.916 & 0.894 \\
\hline $\mathrm{K}^{-} / \pi^{-}$ & $0.15(2)[\mathrm{S}]$ & 0.183 & 0.168 & 0.168 & 0.179 & 0.145 \\
\hline$\overline{\mathrm{p}} / \pi^{-}$ & $0.08(1)[S]$ & 0.082 & 0.084 & 0.078 & 0.083 & 0.078 \\
\hline$\overline{\mathrm{K}_{0}^{*}} / \mathrm{h}^{-}$ & $0.058(17)[\mathrm{S}]$ & 0.055 & 0.049 & 0.049 & 0.046 & 0.032 \\
\hline $\mathrm{K}_{0}^{*} / \mathrm{h}^{-}$ & $0.060(17)[\mathrm{S}]$ & 0.049 & 0.044 & 0.044 & 0.041 & 0.037 \\
\hline
\end{tabular}

Data and BMRS-fit taken from [11].

compared to those obtained in other ideal gas calculations: $T=174 \mathrm{MeV}, \mu_{B}=46 \mathrm{MeV}$ in [11], $T=165 \mathrm{MeV}, \mu_{B}=41 \mathrm{MeV}$ in [30] and $T=190 \mathrm{MeV}, \mu_{B}=45 \mathrm{MeV}$ in [31]. The crossover case in the interacting chiral model (CI) yields $T=170.8 \mathrm{MeV}, \mu_{B}=48.3 \mathrm{MeV}$. Very strong deviations are found for the models with a first-order phase transition (CII, CIII): the freeze-out temperatures are $T=155 \mathrm{MeV}(\mathrm{CII})$ and $T=153.3 \mathrm{MeV}$ (CIII), more than $30 \mathrm{MeV}$ lower than for $i g_{F F M}$. The fitted baryon chemical potentials $\mu_{B}$ increase by about 7-10 MeV. These $T-\mu_{B}$ pairs are very close to the phase boundary (CII) or even right on it (CIII) and are about $10 \mathrm{MeV}$ higher than the values obtained at SPS-energies [32]. Mainly due to the different freeze-out temperatures the values of the corresponding thermodynamic quantities vary between the different approaches. However, the energy per particle $E / A$ is approximately $1 \mathrm{GeV}$ in all cases. This 'unified freeze-out condition' has already been proposed in [33].

The fact that the freeze-out appears right at the phase boundary or at crossover implies that there are large in-medium corrections, in particular for the effective masses, a phenomenon observed already in [17]. The effective masses shown in figure 3 are shifted up to $15 \%$ from their vacuum values. However, all the interacting models show similar values for the effective mass of a given hadron. The strongest in-medium modifications are observed for the non-strange baryons $\left(\Delta m_{i}^{*} / m_{i} \approx 15 \%\right)$. Mesons and strange baryons show smaller changes of the effective masses, e.g. about $10 \%$ for $\Lambda, \pi, \mathrm{K}^{*}$, about $5 \%$ for the kaons and nearly no change for the $\Xi s$.

These results, together with the steep $\chi^{2}$ contours from figure 2 , suggest that the relative particle abundances 'freeze' shortly after the spontaneous breaking of chiral symmetry. 


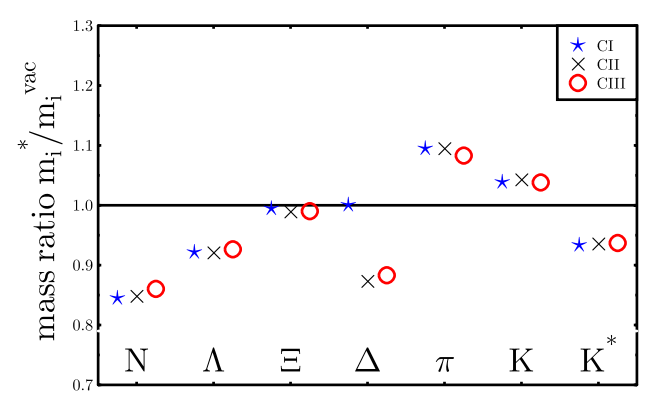

Figure 3. Effective masses for the different interacting chiral models and the ideal gas (vacuum values) case. The differences among the interacting approaches are less than $2 \%$.

The success of our fit suggests extremely rapid chemical equilibration (through abundancechanging reactions) in the state with broken symmetry. Figure 2 shows that the chemical composition of the hadronic system has to change substantially within a small temperature interval, just before freeze-out, even for the crossover transition (i.e. parameter set CI); for reference, we have indicated the dynamical path in the $T-\mu_{B}$ plane corresponding to the expansion of a perfect fluid (i.e. with constant entropy per net baryon [34]). While $2 \rightarrow n$ reactions are perhaps too slow to explain such rapid chemical equilibration $[35,36], m \rightarrow n$ processes with several particles in the initial state may also be important [37-40].

Alternatively, the appearence of chemical equilibrium right after the phase transition (or the crossover) to the state of broken chiral symmetry might just be the outcome of the dynamical symmetry breaking process itself [41], with statistical occupation of the various hadronic channels according to phase space [42-45]. If so, number-changing reactions in the broken phase need not proceed at a high rate. To test this picture experimentally, it might be useful to consider central collisions of small ions like protons or deuterons, at similar energy and particle densities in the central region as for central $\mathrm{Au}+\mathrm{Au}$. For systems of transverse extent comparable to the correlation lengths of the chiral condensates, the dynamical symmetry breaking process should be different from that in large systems (for example, the mean-field approximation should not apply). The correlation lengths $\xi_{\sigma, \zeta}$ are given by

$$
\xi_{\sigma}^{-2}=\frac{\partial^{2}(\Omega / V)}{\partial \sigma^{2}}
$$

and accordingly for $\xi_{\zeta}$. We evaluate the curvature of the thermodynamical potential at the global minimum and for $T, \mu_{B}, \mu_{S}$ at the freeze-out point. For parameter sets CI, CII, CIII we obtain $\xi_{\sigma}=0.37 \mathrm{fm}, 0.41 \mathrm{fm}, 0.40 \mathrm{fm}$, respectively. For the correlation length of the strange condensate we obtain $\xi_{\zeta}=0.20 \mathrm{fm}$ in all three cases. The correlation lengths are not very much smaller than, say, the radius of a proton. Thus, even if the freeze-out point for high-energy $p p$ collisions happens to be close to that for $\mathrm{Au}+\mathrm{Au}$ collisions at RHIC energies, the transition from the symmetry restored to the broken phase might be different. Finally, we also note that the correlation lengths obtained from our effective potential are not larger than the thermal correlation length $1 / T$ at freeze-out, and so corrections beyond the mean-field approximation employed here should be analysed in the future.

\section{Particle ratios from AGS to RHIC}

To obtain the so-called 'freeze-out' curve in the $T-\mu$ plane, the best fits from AGS to RHIC energies are determined, figure 4 shows the deduced temperatures and chemical potentials for 


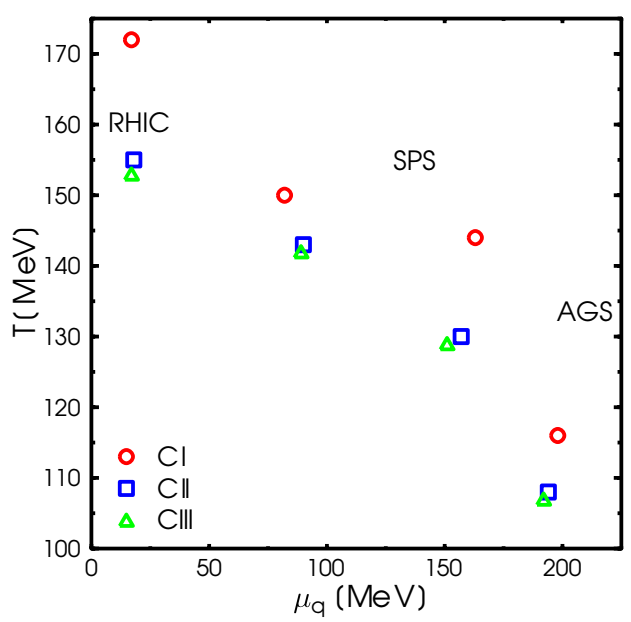

Figure 4. Freeze-out curve in the chiral model (CI, CII, CIII) for relativistic heavy-ion collisions from AGS to RHIC.

the different systems and energies ${ }^{5}$. First, one observes that they are never above the phase transition line (or its generalization for the crossover case, respectively). As already discussed above, for RHIC the deduced freeze-out occurs right at the phase transition border. For SPS $160 \mathrm{GeV}$ the freeze-out is slightly below the phase transition or crossover, respectively and for lower energies further below. Thus, the chemically equilibrated hadron gas phase disappears with increasing energy. As already observed for RHIC, the deduced temperatures for the crossover case (CI) are always considerably above the values obtained in the two scenarios with a first-order phase transition (CII, CIII), while the $\mu_{q}$ values are nearly identical in all cases. This is also illustrated in figure 5, showing the temperature (left) and the chemical potential (right) as a function of centre of mass energy $\sqrt{s}$. Furthermore, one observes that with decreasing energy, the freeze-out temperature decreases and the freeze-out chemical potential increases, in accordance with the results from ideal gas calculations. Comparing the interacting and the non-interacting case (i.e., the freeze-out curve shown in [46]), it turns out that the freeze out values for CI are very close to those obtained in the ideal gas case. In contrast, for CII and CIII considerably lower temperatures are extracted. This shows the uncertainty and the model dependence in the determination of the assumed freeze-out conditions. In general on can state that the stronger the phase transition for $\mu=0$ in the chiral model, the more the freeze-out temperature is lower compared to the ideal-gas case.

In figure 6 the predicted 'freeze-out masses' are depicted as a function of $\sqrt{s}$. They differ by up to about $15 \%$ from their vacuum values. The biggest effect is observed for the non-strange particles, in particular the nucleon and the pion. However, no simple functional dependence on the centre of mass energy $\sqrt{s}$ is observed. Here again further studies are necessary to understand this behaviour, which might also result from the different sets of data available. Figure 6 (right) shows the resulting $\chi^{2}$ values for the different collision systems and energies. It can be seen that all three scenarios give a reasonable description of the data at all energies. If the 'quality' of the fit is measured by the $\chi^{2}$ value, all three parametrizations

5 The investigation for $80 \mathrm{~A} \mathrm{GeV}$ at SPS is not presented here, since not enough data for a reasonable fitting procedure were available. 

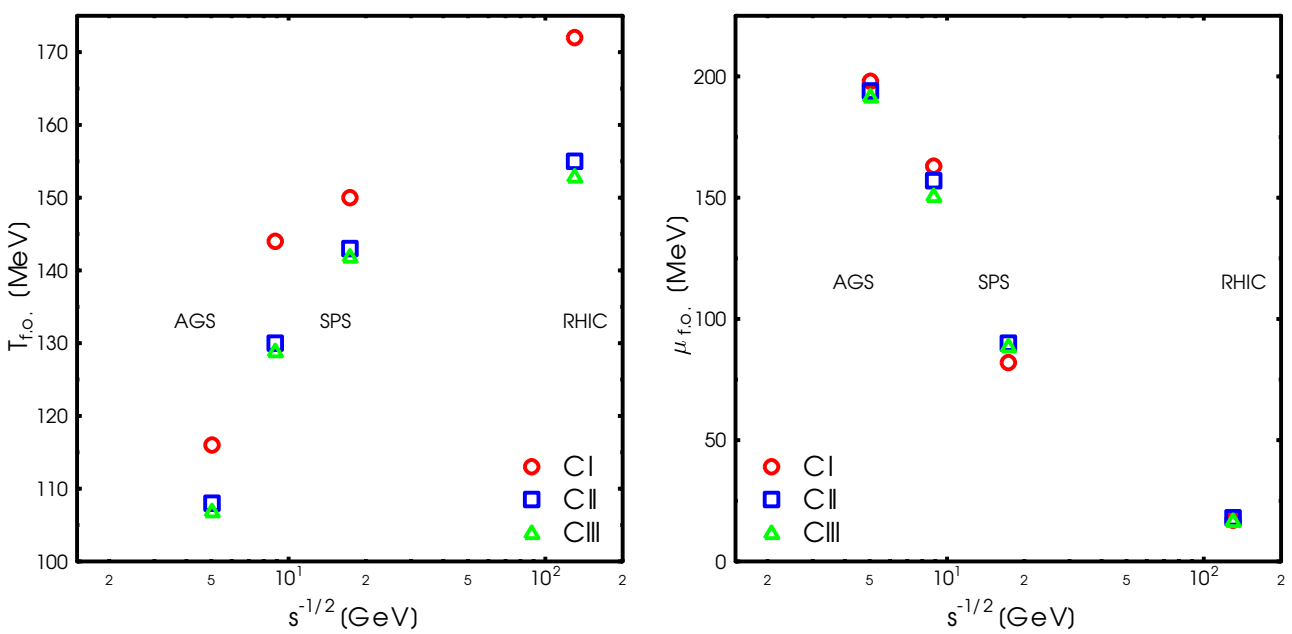

Figure 5. Freeze-out temperature (left) and chemical potential (right) as a function of $\sqrt{s}$ for CI, CII and CIII.
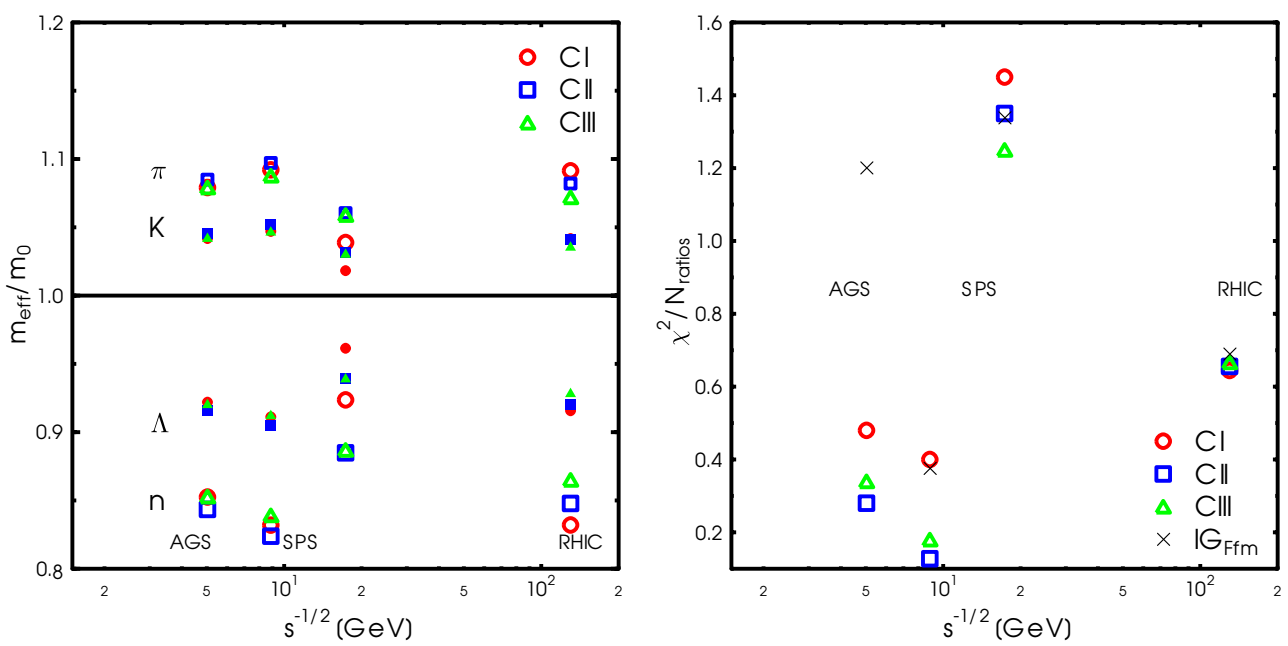

Figure 6. Effective masses of $\mathrm{N}, \Lambda, \pi, \mathrm{K}$ (left) and $\chi^{2}$ value (right) as a function of $\sqrt{s}$ for CI, CII and CIII.

are equally 'successful' in describing the data. The differences in the $\chi^{2}$ values are too small to discriminate between the different scenarios.

\section{Conclusion}

Particle ratios as calculated in a chiral $S U(3) \sigma-\omega$ model are compared with data for A-A collisions from AGS to RHIC. and with non-interacting gas calculations. Since different versions of the chiral model show qualitatively different phase transition scenarios, we investigate whether the particle production, i.e. the chemistry of the system, is sensitive to the phase transition behaviour. Since we have shown that the current data are described by 
all three different phase transition scenarios and the ideal gas model, we can so far not favour or rule out any one scenario.

In all interacting models the effective masses at freeze-out are shifted up to $15 \%$ from their vacuum values. The fitted chemical freeze-out temperatures and chemical potentials considerably depend on the order of the phase transition. Furthermore, at RHIC the fitted freeze-out points are located practically right on the phase transition boundary (at SPS slightly below it), in the first-order phase transition scenarios, but $T$ is always $\leqslant T_{c}$. This suggests that at RHIC the system emerges after the chiral-chiral phase transition. This of course is only true if a first-order phase transition does actually occur in QCD at small chemical potentials and high $T$. 'Freezing' of the relative abundances of various hadrons in the symmetric phase (at $T>T_{c}$ ) is excluded.

\section{Acknowledgments}

The authors are grateful to A Dumitru, J Schaffner-Bielich and I Mishustin for fruitful discussions. This work is supported by Deutsche Forschungsgemeinschaft (DFG), Gesellschaft für Schwerionenforschung (GSI), Bundesministerium für Bildung und Forschung (BMBF), the Graduiertenkolleg Theoretische und Experimentelle Schwerionenphysik and by the US Department of Energy, Nuclear Physics Division (Contract no W-31-109-Eng-38). This work used computational resources provided by the the BCPL Bergen, Norway and the Center for Scientific Computing (CSC) at the University of Frankfurt, Germany.

\section{References}

[1] Fermi E 1950 Prog. Theor. Phys. 5570

[2] Landau L D 1953 Izv. Akad. Nauk SSSR Ser. Fiz. 1751

[3] Westfall G D et al 1976 Phys. Rev. Lett. 371202

[4] Stöcker H, Greiner W and Scheid W 1978 Z. Phys. A 286121

[5] Csernai L P and Kapusta J I 1986 Phys. Rep. 131223

[6] Hahn D and Stöcker H 1986 Nucl. Phys. A 452723

[7] Braun-Munzinger P, Stachel J, Wessels J P and Xu N 1996 Phys. Lett. B 3651

[8] Letessier J and Rafelski J 2000 Int. J. Mod. Phys. E 9107

[9] Rafelski J, Letessier J and Torrieri G 2001 Phys. Rev. C 64054907

[10] Becattini F et al 2001 Phys. Rev. C 64024901

[11] Braun-Munzinger P, Magestro D, Redlich K and Stachel J 2001 Phys. Lett. B 51841

[12] Braun-Munzinger P and Stachel J 1998 Nucl. Phys. A 6383

[13] Cleymans J and Redlich K 1999 Phys. Rev. C 60054908

[14] Papazoglou P et al 1998 Phys. Rev. C 572576

[15] Papazoglou P et al 1999 Phys. Rev. C 59411

[16] Zschiesche D et al 2000 Springer Tracts Mod. Phys. 163129

[17] Theis J et al 1983 Phys. Rev. D 282286

[18] Schaffner J et al 1991 Z. Phys. A 34147

[19] Brown G E and Rho M 1996 Phys. Rep. 269333

[20] Michalec M, Florkowski W and Broniowski W 2001 Phys. Lett. B 520213

[21] Zschiesche D, Schramm S, Stocker H and Greiner W 2002 Phys. Rev. C 65064902

[22] Karsch F, Laermann E and Peikert A 2001 Nucl. Phys. B 605579

[23] Karsch F 1999 Preprint hep-lat/9903031

[24] Serot B D and Walecka J D 1997 Int. J. Mod. Phys. E 6515

[25] Schechter J 1980 Phys. Rev. D 213393

[26] Zschiesche D et al 2001 Nucl. Phys. A 68134

[27] Bass S A et al 1998 Prog. Part. Nucl. Phys. 41225

[28] UrQMD Collaboration http://de www th physik uni-frankfurt/urqmd/

[29] Michalec M 2001 PhD Thesis Institute of Nuclear Physics, Crakow 
[30] Florkowski W, Broniowski W and Michalec M 2002 Acta Phys. Pol. B 33761

[31] Xu N and Kaneta M 2002 Nucl. Phys. A 698306

[32] Zschiesche D et al unpublished

[33] Cleymans J and Redlich K 1998 Phys. Rev. Lett. 815284

[34] Subramanian P R, Stocker H and Greiner W 1986 Phys. Lett. B 173468

[35] Bass S A, Danielewicz P, Pratt S and Dumitru A 2001 J. Phys. G: Nucl. Part. Phys. 27635

[36] Bass S A and Dumitru A 2000 Phys. Rev. C 61064909

[37] Rapp R and Shuryak E V 2001 Phys. Rev. Lett. 862980

[38] Greiner C and Leupold S 2001 J. Phys. G: Nucl. Part. Phys. 27 L95

[39] Greiner C 2002 J. Phys. G: Nucl. Part. Phys.. 281631

[40] Greiner C 2002 Nucl. Phys. A 698591

[41] Scavenius O, Dumitru A and Lenaghan J T 2002 Preprint hep-ph/0201079

[42] Becattini F and Heinz U W 1997 Z. Phys. C 76269

[43] Becattini F, Gazdzicki M and Sollfrank J 1998 Nucl. Phys. A 638403

[44] Stock R 1999 Nucl. Phys. A 661282

[45] Stock R 1999 Phys. Lett. B 456277

[46] Braun-Munzinger P, Redlich K and Stachel J 2003 Preprint nucl-th/0304013 